\title{
Article \\ A conceptual model for the origin of the cutoff parameter in Exotic Compact Objects
}

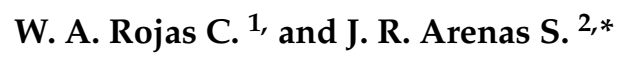 \\ 1 Departamento de Física , Universidad Nacional de Colombia, Email: warojasc@unal.edu.co \\ 2 Observatorio Astronómico Nacional, Universidad Nacional de Colombia, Email: jrarenass@unal.edu.co
}

\begin{abstract}
We propose a conceptual model for the closeness parameter $\epsilon$, which characterizes exotic compact objects (ECOs). To estimate $\epsilon$, a thin spherical dust shell is considered, which gravitationally contracts from a specific position $r\left(t_{0}\right)$ to near its gravitational radius $r\left(t_{2}\right)=r_{s}+\epsilon$, in a finite time $t_{2}$, measured in the frame of a fiducial observer (FIDO). For an external observer, the shell's kinematics is characterized by two clearly distinguishable phases: one of rapid contraction, where the shell is far away from the gravitational radius, $r\left(t_{0}\right) \gg r_{s}$, and a second phase quasi-stationary, $r(t) \sim r_{s}$, where all of the shell's mass is concentrated around the associated horizon, such that for a FIDO, a black hole (BH)is undistinguishable from a shell configured as a black shell (BS).

In the semi-classical approximation $E \ll \kappa_{0} l_{p}^{2}$ and tends to zero when the observation time of collapse $t_{2}$, measured by FIDO, tends to infinity; $\kappa_{0}$ and $l_{p}$ are surface gravity and Planck length, respectively. The quantum effects are significant when $\epsilon \ll r\left(t_{2}\right)$ and $\epsilon$ tends to $\kappa_{0} l_{p}^{2}$.

Without knowing details on quantum gravity, parameter $\epsilon$ is calculated, which, in general, allows distinguishing the ECOs from BHs. Specifically, a BS (ECO) is undistinguishable from a BH.
\end{abstract}

Keywords: black shell, closeness parameter, cutoff, entanglement, exotic objects, compact objects

PACS: 04.,04.20-q,04.70Bw,05.20.Gg

\section{Introduction}

Experimental evidence of gravitational radiation (GW) have been found for compact objects [1-3], including the event GW190814, where the nature of a compact object is unknown and could be an exotic compact object [4]. This has established a new field of observational astronomy, i.e., gravitational waves astronomy, which enables the study of binary systems, such as ECO-ECO, BH-BH, and ECO-BH.

Abedi et al. analyzed the gravitational waves GW150914, GW151226, and LVT151012; then they suggested that GW signals arise due to merging BHs or ECOs systems [5]. In addition, Cardoso et al. showed that in some configurations the coalescence of ECOs (compact boson stars) might be almost indistinguishable from those of $\mathrm{BHs}[6]$.

ECOs are of great importance, not only in research about the nature and existence of astrophysics BHs, but also the parameter $\epsilon$ that characterized them would be associated with some basic scale of quantum gravity [7]. The observation of coalescence of compact objects based on the study of gravitational radiation would aid in explaining the nature of quantum gravity. ECOs are inspired on the quantum effects of BHs and seek to provide an answer to BH problems, such as information loss and the singularity [8].

An ECO can be characterized by the closeness parameter

$$
\epsilon=r-r_{s}
$$

for $r \sim r_{s}$, where $r$ describes the position of surface of the ECO and $r_{s}=\frac{2 G M}{c^{2}}$ is the radius associated to the event horizon [7,9]. This parameter allows quantifying how compact an ECO [10].

$\epsilon$ is a quantity depending on the observer that is associated to the Love numbers [7] 


$$
\epsilon=r_{s} e^{-1 / k}
$$

where $k$ is one of the Love numbers. Hence, $\epsilon$ would allow distinguishing a $\mathrm{BH}$ from an ECO in general, but it would also show that a BH would be undistinguishable from a BS.

In Newtonian Physics, Love numbers relate the mass multipole moments created by tidal forces on a spherical body. These moments encode information on the body's internal structure and it can be transported by the GWs. In other words, the deformity of an ECO induced by tidal forces is coded by Love numbers and sensitively depend on the ECO's internal structure. Determining Love numbers implies understanding physics of the ECO [11].

In the case of an ECO, the Love numbers are different from zero and are encoded in the gravitational waves, in binary ECOs, they allow studying the behavior of matter with enormous densities and for a static BH kind as Schwarzschild, they are null, which leads to the multipole structure remaining undisturbed when immerred in a tidal field [12,13]. In 2017, Cardoso et al. determined that a Schwarzschild BH in Chern-Simons gravity has Love numbers different from zero [14]. We expect that by characterizing the ECOs through Love numbers, the Love relationships contained in the GWs from the fusion of binary systems will allow to understand the behavior of the matter subjected to enormous densities and the physics of the GWs themselves $[13,15]$. Recently, Le Tiec et al. estimated the Love numbers for a Kerr BH, which would imply that they would be susceptible to the deformity of an external tidal field and that such deformity could be detected in GW-Ligo [16].

There are several conceptions of the parameter $\epsilon$. Among others, Guo et al. obtained, for one type of ECO referred to as fuzzball $\epsilon \ll G M$ close to $r_{s}$, and for a firewall $\epsilon \sim l_{p}^{2} / G M$ [17]. It is interesting to mention the results with significant quantum effects such as [8]

$$
\epsilon=\frac{t_{P}}{t_{H}}=10^{-39}-10^{-46}, t_{P}=\sqrt{\frac{\hbar G}{c^{5}}}, t_{H}=\frac{G M}{c^{3}} .
$$

Also, for an ECO (BS), a model is introduced that associates the creation of particles and a closeness parameter [18]

$$
\epsilon=\sqrt{1-\frac{2 M}{r_{f}}} \ll 1,
$$

where $r_{f}$ is the radius limit of approach.

The instability of the ECOs and their implications in the GWs are proposed by B. Chen et al., where an ECO has a radius

$$
r_{E C O}=r s+\epsilon, \Delta=\sqrt{8 M \epsilon} .
$$

Where $\Delta$ is a supplementary characterization of the compactness of the ECO [10]. Additionally, it has been shown that ECOs are unstable due to the accretion of matter and to the influence of GWs on gravitational collapse, resulting in when $\epsilon \sim l_{p}$, they necessarily convert into BHs. To prevent gravitational collapse, $\epsilon \gg l_{p}$ must be met or the null conditions of energy are violated[19].

Black shells share properties of radiation emission with the BHs when they are considered quantum fields in the vicinities of the BSs. Any shell of a mass $M$ that collapses until close to its gravitational radius, emits thermal radiation, with a radius limit given by [20]

$$
r_{f}=\frac{2 M}{1-\epsilon^{2}} .
$$

In the case of a closeness parameter $\epsilon \longrightarrow 0$, a BS possesses properties analogous to a $\mathrm{BH}$, such as radiation at the Hawking temperature $T_{H}$ and a Berkenstein-Hawking entropy $S_{B H}$ [21,22]. For other types of ECOS, stability differs, for example, gravastar ECOs are more thermodynamically stable in contrast to the BHs [23]. 
Within the wide spectrum of ECOs registered in detail in [9], which includes from white dwarfs and neutron stars, to exotic objects such as quark stars, hybrid stars with gluon-plasma nuclei, superspinars, wormholes, etc., the gravastars (GS) and the boson stars (BSt) stand out, which as the black shells, are feasible alternatives to astrophysical BHs, but the GS and BSt are unstable since they exhibit a very unstable ergoregion and has a very short life [24].

Research on the nature of the ECOs goes beyond theoretical speculations because its study is being developed with models thought for observation. It is important to know the available observational methods to complete the compact object search scenario. A usual method to distinguish a neutron star from a $\mathrm{BH}$ is based on measuring their mass. In the case of an ECO, if its mass is greater than the Chandrasekhar limit, it is believed that the ECO is a BH. Nevertheless, luminosity criteria associated to mass measurements and angular momentum are not entirely reliable methods given the broad spectrum of ECOs that could be found, which is why other techniques to distinguish them are needed. A promising technique to identify ECOs from BHs lies in the observation of GWs. It is thought that the study of GWs in the inspiral phase of the fusion of binary systems allows determining their mass and the multipole moments distinguishing an ECO from a BH [25]. In 2016, Cardoso et al. studied the GWs product of the fusion of two BSs of equal mass and they compared them to the GWs corresponding to two BHs. They found that under certain configuration the GW signals are almost undistinguishable [6]. Another method to distinguish ECOs from BHs was proposed by Cardoso et al., where they consider that compact objects with rings of light are $\mathrm{BHs}$, given that the light rings are associated to the photon sphere at a radius $r=3 M$, in contrast to the ECOs that do not exhibit these luminous rings[25] . Current evidence leads to think that ECOs with high angular momentums are similar to a Kerr BH but without the formation of event horizons [26].

Based on this conceptualization of the ECOs in the observational context described, our main purpose in this paper is to contribute to the characterization of the nature of the ECOs in terms of the closeness parameter $\epsilon$. We propose a model that relates criteria from the quantum microscopic world of compact objects with observations corresponding to the astrophysics macroscopic world, as a basis for estimating $\epsilon$.

With relativistic kinematic properties, referring to the observer's notion, we model a type of ECO referred to as black shell, undistinguishable from a $\mathrm{BH}$, and that is an alternative object to the $\mathrm{BHs}$, as it would be observed by a FIDO.

$\epsilon$ was calculated based on quantum foundations that establish a connection with the notions of quantum gravity through of the Berkenstein-Hawking entropy and which exhibits a semi-classic and another limit where the quantum effects are significant.

$\epsilon$ is a measure of the compactness of the ECOs, which we expect is indicator to classify and distinguish compact objects in general, through the relationship existing between this parameter and the Love numbers.

In Section 2, the quantum origin of the closeness parameter $\epsilon$ that characterizes the ECOs is substantiated. Section 3, describes that kinematic nature of the cutoff parameter for a BS. The quantum model of the closeness parameter is introduced in Section 4 and Section 5 is devoted to summarizing and discussing this paper.

\section{Quantum mechanical foundation of the cutoff for a BS}

Let be a thin spherical dust shell that gravitationally contracts from a certain position $r_{0}$ to near its gravitational radius $r\left(t_{2}\right)=r_{s}+\epsilon$, at a finite time $t_{2}$ measured by a FIDO observer.

The nature of the closeness parameter $\epsilon$ obeys a bound of a quantum nature and is closely related to the shell's entropy compacted close to the gravitational radius.

The origin of this entropy is understood in terms of the properties of the physical vacuum in strong gravitational fields. In the case of $\mathrm{BHs}$, an observer that is at rest with respect to the horizon, sees the zero-point fluctuations of physical fields as a thermal atmosphere around a BH [27]. An 
important property of the BHs is that the entanglement entropy $S_{\text {ent }}$ coincides with the entropy of that thermal atmosphere [28-32].

$S_{\text {ent }}$ may be a source of Berkenstein-Hawking entropy $S_{B H}$ for a species, if $\epsilon \neq 0$ in the expression $[28,29,33]$

$$
S_{\text {ent }} \sim \frac{A}{4 \epsilon}
$$

where $A$ is a surface very close to the event horizon, with its proper altitude $\eta$ related to the parameter $\epsilon$ through the relationship

$$
\epsilon=\frac{1}{2} \frac{\kappa_{0}}{c^{2}} \eta^{2}
$$

and with $\eta^{2} \sim l_{p}^{2}$; being $\kappa_{0}$ the surface gravity and $l_{p}$ the Planck length.

Depending on $\epsilon$, without considering the dependency that $S_{B H}$ would have on all the fields present in nature, in principle, it can be identified with $S_{\text {ent }}$. In generally, the two entropies differ in one constant. What matters is that the surface of the shell collapsing coincides with area $A$ in (7) and that it can be interpreted in the same way as $S_{B H}$.

Thus, the entropy associated to the shell is considered as the thermodynamic entropy of equilibrium. This entropy corresponds to the information stored in the material that comes together to form a black hole, compressed in one thin layer close to the gravitational radius. Since the entropy for a given mass and area is maximized by the thermal equilibrium, we expect it to be the maximum entropy that could be stored in the material before it crosses the horizon [34].

The fact that the entropy of a $\mathrm{BH}$ is also the maximum entropy that may be obtained through the Berkenstein limit was one of the main observations that led to the holographic principle [35,36]. The maximum entropy stored in the shell is related to an upper bound in the information that is understood in terms of quantum bounds that are based on principles of quantum gravity $[7,27,37]$.

In terms of the expression (7), the estimate of $\epsilon$ is introduced, which is calculated from the contribution of Area $S_{A}$ of $S_{\text {ent }}$. This contribution is determined by the quantum condition $[29,33]$

$$
\epsilon \ll \Delta \ll r\left(t_{2}\right),
$$

illustrated in Figure $1, \Delta$ is a measure of the integration region that leads to $S_{A}$.

Calculating $S_{\text {ent }}$, for simplicity, is done based on the scalar field in the vicinities of the shell when it is very close to its gravitational radius, resorting to quantum field theory in a curved spacetime [28,33]. In this article, to perform this estimate, we use the particle description of quantum fields.

\section{Kinetic origin of the cutoff parameter for a bs}

The equation of movement of the shell with mass $M$ in gravitation contraction, described in Section 2, is expressed by [38]

$$
\frac{d R}{d \tau}=\sqrt{\left[a+\frac{M}{2 a R}\right]^{2}-1}
$$

where $a$ is the quotient between $M$ and its mass at rest $m, \tau$ is proper time in the frame of a freely falling observer (FFO).

The FIDO observer that measures the collapse time of the shell is in an asymptotically flat spacetime region around the BS:

$$
d s^{2}=-f(r) d t^{2}+\frac{1}{f(r)} d r^{2}+r^{2} d \theta^{2}+r^{2} \sin ^{2} \theta d \phi^{2}, f(r)=1-\frac{2 G M}{c^{2} r} .
$$

A simple solution to the equation of movement of the shell (10) can be expressed by the function [39-41] 


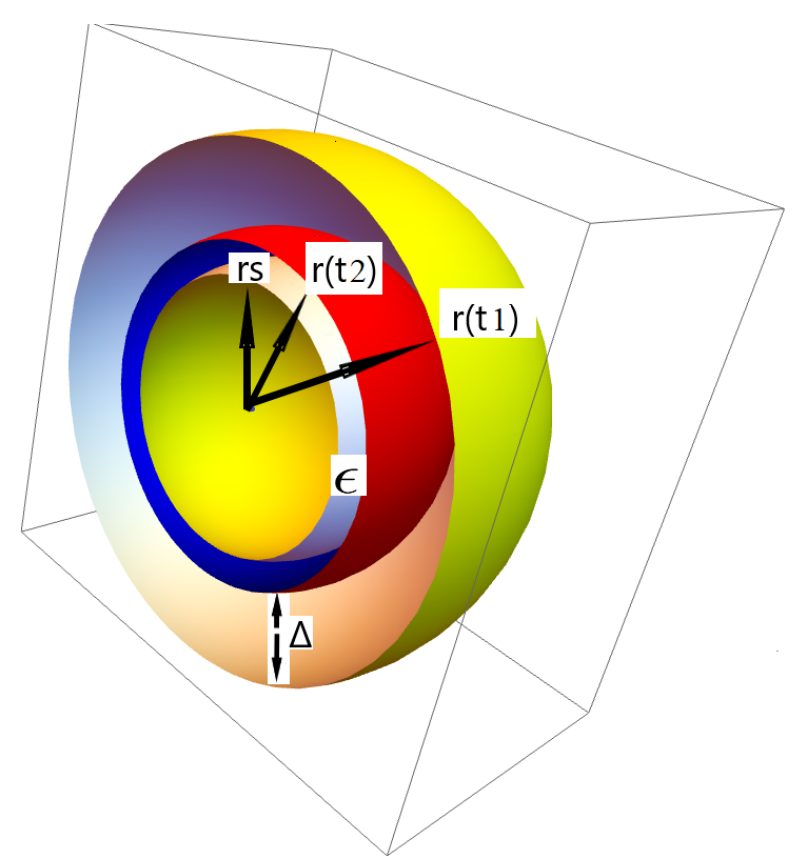

Figure 1. Configuration of the vicinity of the gravitational radius.

$$
r(t)=r_{s}+\delta r e^{-t / \bar{\tau}}
$$

where $\delta r=r_{0}-r_{s}, \bar{\tau}=\frac{4 G M}{3 c^{3}}$ and $r_{s}=\frac{2 G M}{c^{2}}$.

For an external observer, the kinematic of the shell is characterized by two clearly distinguishable phases: one of rapid contraction, where the shell is far away from the gravitational radius, $r\left(t_{0}\right) \gg r_{S}$, and a second quasi-stationary phase in the region where $r(t) \sim r_{s}$, and the shell's mass is concentrated around the associated horizon.

The shell's movement in the first phase of collapsing is classically described based on the general theory of relativity (GTR). The collapsing phase close to the gravitational radius is adjusted introducing quantum effects within the framework of the quantum field theory in curved spacetime, such that

$$
\lim _{t \longrightarrow t_{2}} r(t)=r_{s}+\epsilon
$$

The collapsing phase in the vicinity of the horizon is related to the classic phase according to Figure 2, where the magnification of the vertical segment on the axis $r(t), \overline{r_{s} r\left(t_{1}\right)}$ is shown in Figure 3

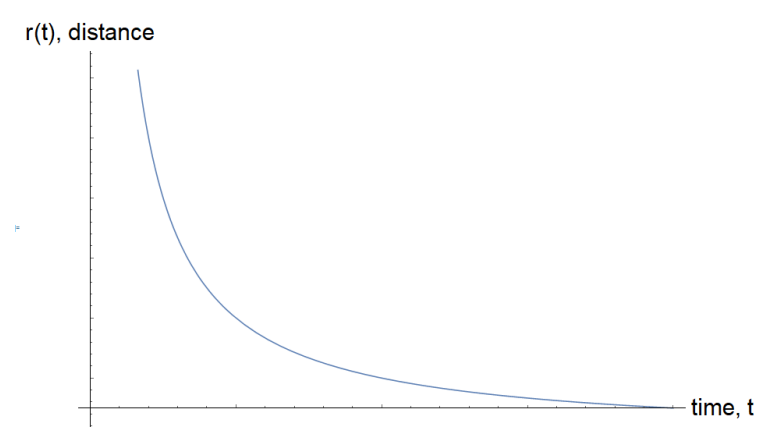

Figure 2. Phase of rapid collapse. 


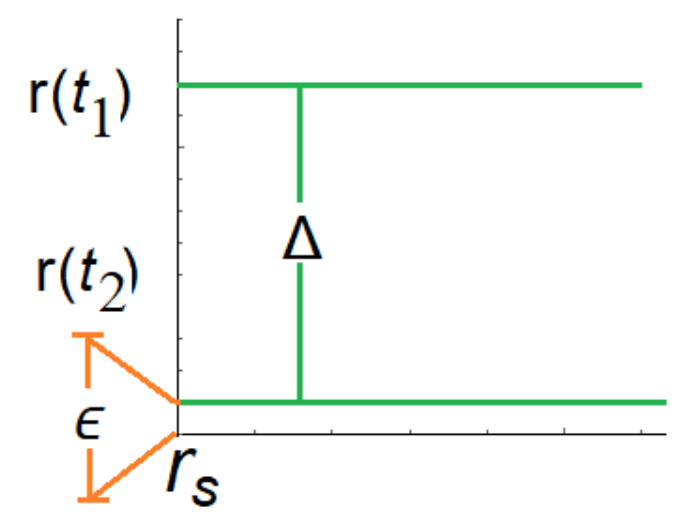

Figure 3. Classical description of the gravitational collapse with a quantum bound.

In the gravitational background given by (11), expression (7) is calculated in terms of the entropy

$$
S=\int_{V} s d V
$$

where $s$ is the entropy density of the thermal atmosphere in the vicinity of the shell, which is expressed as a function of the energy density $\rho$ and pressure $P$ as

$$
s=\beta k_{B}[\rho+P], \quad \beta=\frac{1}{k_{B} T}
$$

where $k_{B}$ is the Boltzmann constant and $T$ is the local temperature given by

$$
T(r)=\frac{T_{\infty}}{\sqrt{f(r(t))}}
$$

for a uniform temperature $T_{\infty}$ in the asymptotically flat region.

In addition, the differential volume $d V$ corresponds to

$$
d V=\frac{4 \pi}{\sqrt{f(r(t))}} r^{2}(t) d r(t)
$$

Entropy (14) has two contributions: one dependent on volume $S_{V}$ for $r \gg r_{s}$ and another one dependent on the area $S_{A}$ for $r \sim r_{S}$ :

$$
S=S_{V}+S_{A}
$$

The entropy density $s$ for the contribution $S_{A}$ is calculated based on (15) resorting to the ultrarelativistic approaches corresponding to high local temperatures, where

$$
P \cong \frac{1}{3} \rho, \rho=\frac{3 T^{4}}{\pi^{2}}
$$

and with which, we obtain $[28,33,42,43]$

$$
S=\frac{16 \pi}{3 h^{3} c^{2} T} \int_{0}^{\infty} \frac{E^{3}}{e^{E / k_{B} T}-\sigma} d E,
$$

where $E$ is energy, $c$ is the speed of light, and $h$ is Planck's constant, with $\sigma=+1$ for bosons and $\sigma=-1$ for fermions. In the model proposed, we take $\sigma=0$.

From the expressions (14) and (20), we obtain 


$$
S_{A}=\frac{16 \pi}{3 h^{3} c^{2}} \int_{V} d V \frac{1}{T} \int_{0}^{\infty} \frac{E^{3}}{e^{E / k_{B} T}} d E .
$$

Then, the magnitude of the volume element, according to (12), can be written as

$$
|d V|=\frac{4 \pi}{\sqrt{f(r(t))}} \frac{r_{s}^{2} \delta r e^{-t / \bar{\tau}}}{\bar{\tau}} d t
$$

where the small summands are disregarded in the expression

$$
|d V|=\frac{4 \pi}{\sqrt{f(r(t))}}\left[\frac{r_{s}^{2} \delta r e^{-t / \bar{\tau}}}{\bar{\tau}}+\frac{2 r_{s} \delta r^{2} e^{-2 t / \bar{\tau}}}{\bar{\tau}}+\frac{\delta r^{3} e^{-3 t / \bar{\tau}}}{\bar{\tau}}\right] d t,
$$

Since $e^{-t / \bar{\tau}} \gg e^{-2 t / \bar{\tau}} \gg e^{-3 t / \bar{\tau}}$. (23)

Substituting (20) and (22) into (14),

$$
S_{A}=\frac{16 \pi}{3 h^{3} c^{3}} \int \frac{4 \pi}{\sqrt{f(r(t))}} \frac{r_{s}^{2} \delta r e^{-t / \bar{\tau}}}{\bar{\tau}} d t \frac{1}{\tau} \int_{0}^{\infty} \frac{E^{3}}{e^{E / k_{B} T}} d E
$$

Finally, based on the local temperature (16), $S_{A}$ is expressed

$$
S_{A}=\frac{32 k_{B}^{4} c}{h^{3}}\left(\frac{T_{\infty}^{3}}{\kappa_{0}^{2}}\right) \frac{A_{H} C_{1}}{4 \delta r}
$$

where $A=4 \pi r_{s}^{2}$ and we introduce the definition of

$$
C_{1}=\frac{1}{\bar{\tau}} \int_{t_{1}}^{t_{2}} e^{t / \bar{\tau}} d t
$$

corresponding to the collapse time between $r\left(t_{1}\right)$ and $r\left(t_{2}\right)$ in the configuration of Figure 1.

The expression (25) corresponds to the known expression (7), where $t_{1}$ determines the finite nature of entropy in temporal terms, in contrast to $\epsilon$, which in (7), does the same thing in spatial terms.

From (12) and (26), we obtain a relationship between the times measured by the FIDO observer and the closeness parameter $\epsilon$ as modelled in Section 4.

\section{Quantum model of the closeness parameter}

From the expressions (12) and (26):

$$
-\int_{r\left(t_{1}\right)}^{r\left(t_{2}\right)} \frac{\delta r}{\left(r-r_{s}\right)^{2}} d r=\frac{1}{\bar{\tau}} \int_{t_{1}}^{t_{2}} e^{t / \bar{\tau}} d t
$$

where $r\left(t_{1}\right)=r\left(t_{2}\right)+\Delta$ and $r\left(t_{2}\right)=r_{s}+\epsilon$.

From integrating (27), we obtain

$$
\delta r\left[\frac{1}{\epsilon}-\frac{1}{\epsilon+\Delta}\right]=e^{t_{2} / \bar{\tau}}-e^{t_{1} / \bar{\tau}} .
$$

According to (9), $\epsilon \ll \Delta$. Then,

$$
\begin{gathered}
\delta r\left[\frac{1}{\epsilon}-\frac{1}{\Delta}\right]=e^{t_{2} / \bar{\tau}}-e^{t_{1} / \bar{\tau}} . \\
\delta r\left[\frac{\Delta-\epsilon}{\Delta \epsilon}\right] \sim\left[\frac{\Delta}{\epsilon \Delta}\right] \delta r=e^{t_{2} / \bar{\tau}}-e^{t_{1} / \bar{\tau}} \\
\epsilon=\frac{\delta r}{e^{t_{2} / \bar{\tau}}-e^{t_{1} / \bar{\tau}}} .
\end{gathered}
$$


The classical expression (12) can be quantum-adjusted considering the following:

We expect that (12), at the limit when $t \longrightarrow t_{2}$, according to the criterion (9), is like the limit described by (13). In other words, according to (13) and (28):

$$
r\left(t_{2}\right)=r_{s}+\frac{\delta r}{e^{t_{2} / \bar{\tau}}-e^{t_{1} / \bar{\tau}^{\prime}}}
$$

which can be taken as a starting expression to generalize expression(12), setting the value for $t_{1}$ and leaving $t_{2}$ as a variable:

$$
r(t)=r_{s}+\frac{\delta r}{e^{t / \bar{\tau}}-e^{t_{1} / \bar{\tau}}}
$$

for which, it is required that $\mathrm{t}$ is finite and $t>t_{1}$, corresponding to the time of collapse measured by the FIDO.

From (30), it is possible to obtain a semi-classic limit if $t_{2} \gg t_{1}$ :

$$
\epsilon \sim \delta r e^{-t_{2} / \bar{\tau}}
$$

where for the distant FIDO observer, we expect that

$$
\epsilon \ll \kappa_{0} l_{P}^{2}
$$

The latter expression has a quantum origin due to the relationship that exists between $t$ and $\epsilon$ given by (27), provided $t_{2}$ is finite. A totally classic result occurs when $t_{2}$ tends continuously to infinity and consequently, $\epsilon$ tends to zero.

$t_{1}$ can be estimated considering that $r\left(t_{2}\right) \ll r_{0}$ and that $r\left(t_{2}\right)=r_{s}+\epsilon \sim r_{s}$.

From (12) and

$$
\Delta=r\left(t_{1}\right) \breve{r}\left(t_{2}\right)
$$

so:

$$
\begin{gathered}
\frac{\Delta}{\delta r}=e^{-t_{1} / \bar{\tau}}-e^{-t_{2} / \bar{\tau}} \\
\frac{\Delta}{r_{0}-r_{s}} \sim \frac{r_{s}^{2}}{r_{0}^{2}}=e^{-t_{1} / \bar{\tau}}-e^{-t_{2} / \bar{\tau}}
\end{gathered}
$$

where

$$
\frac{\Delta}{r\left(t_{2}\right)} \sim \frac{r\left(t_{2}\right)}{r_{0}}, \frac{\Delta}{r_{s}} \sim \frac{r_{s}}{r_{0}} \Rightarrow \Delta \sim \frac{r_{s}^{2}}{r_{0}}
$$

(28) can be written

$$
\epsilon=\frac{\delta r}{D e^{\left(t_{1}+t_{2}\right) / \bar{\tau}}}
$$

with $D=\frac{r_{s}^{2}}{r_{0}^{2}}$.

At the limit $t_{1} \ll t_{2}$, (34) must coincide with (31). Comparing these las two expressions we obtain

$$
e^{t_{1} / \bar{\tau}}=\frac{r_{0}^{2}}{r_{s}^{2}}
$$

Then, from (28) and (35)

$$
\epsilon=\frac{\delta r}{e^{t_{2} / \bar{\tau}}-\frac{r_{0}^{2}}{r_{s}^{2}}} .
$$


In (36), we included a preliminary criterion given by (33) that can be adjusted observationally in such a way that $\epsilon$ expressed by (36), which is clearly greater than its corresponding magnitude given by (31), approaches $\kappa_{0} l_{p}^{2}$.

\section{Summary and Discussion}

ECOs have become very important objects of research on the nature and existence of astrophysics BHs, considering that these objects are not ruled out by the recent gravitational wave observations [4]. In addition, the closeness parameter $\epsilon$ that characterizes the ECOs would be associated to any basic scale of quantum gravity [7]. In that context, this paper contributes to the conceptualization of the origin of the cutoff parameter $\epsilon$, with a model that characterizes the ECOs according to the frame of the observer [44].

The model proposed consists of a thin spherical dust shell that gravitationally contracts from a specific position $r_{0}$ to close to its gravitational radius $r\left(t_{2}\right)=r_{s}+\epsilon$, at a finite time $t_{2}$ measured in the frame of a FIDO.

Based on the bound that determines the maximum information that the shell can store compressed in a thin layer close to the gravitation radius, we estimate $\epsilon \ll \kappa_{0} l_{p}^{2}$ in a semi-classical approach and a limit of $\epsilon$ tending to $\kappa_{0} l_{p}^{2}$ when the quantum effects are significant.

The maximum information associated to the shell is quantified with the contribution of area $S_{A}$ to its entropy [34]. With this expression, we introduce a suggestive relationship between the microscopic world and the macroscopic world.

Usually $S_{A}$ is calculated in a no divergent manner in terms of a cutoff $\epsilon$, as shown in expression (7), and it is adjusted with a quantum gravity criterion to calculate the Berkenstein-Hawking entropy $S_{B H}$. In this article, we propose a divergence control in terms of a finite time $t_{2}$, according to the definition of $C_{1}$ in (26). What is interesting is that the two criteria are related through the expression (27) and more specifically, based on (36). In other words, since the entropy $S_{B H}$ is proportional to the shell's entropy at the limit when $r(t) \sim r_{s}$, astrophysical measurements of $t_{2}$ and $r_{0}$, would allow estimating the cutoff $\epsilon$. With this relationship, we have an observational criterion to compare the assumptions made based on the principles of quantum gravity.

On the other hand, the estimates of $\epsilon$ can be compared with those respectively calculated in articles reported in $[8,17,18]$ and also related to the parameters of the different types of ECOs through the relationship of $\epsilon$ with the Love numbers [7].

The ECO model being propose depends on external observation and is compatible with $\mathrm{BHs}$ in two senses: with respect to the FIDO observers, this type of ECO is undistinguishable from a $\mathrm{BH}$, and regarding a FFO observer, the shell collapses into a $\mathrm{BH}$. In this latter case, the explanation of $S_{B H}$ requires a complete theory of quantum gravity. In the case of the description in terms of the external observers at rest with respect to the associated horizon, without knowing details about quantum gravity, it is possible to interpret $S_{B H}$ as a thermodynamic entropy.

$\mathrm{BH}$ formation, maturity, and death are still not completely understood, total understanding may establish the existence of a quantum gravity theory. Specifically, a BH is characterized by a singularity surrounded by an event horizon, which hides the interior of the BH from fiducial observers (FIDOs) $[45,46]$. This is key because it distinguishes a BH from other types of ECOs in the frame of a FIDO. This implies that the geometry outside the ECO is consistent with that of a $\mathrm{BH}$ [9].

It is noteworthy to mention that the role of the FIDO is remarkable in this study since the cutoff parameter is only measured by such observers [7,9].

This model can be improved if a dynamic spacetime, such as a Kerr spacetime, is considered because the cutoff parameter in equation (12) takes into account observational data [47,48]. 


\section{Acknowledgments:}

This work was supported by the Departamento de Administrativo de Ciencia, Tecnología e Innovación, Colciencias.

\section{Conflicts of Interest:}

The authors declare no conflict of interest.

\section{References}

1. B.P. Abbott et al. Phys. Rev. Lett., 116, 061102, 2016.

2. B. P. Abbott et al. Astrophys. J. Lett., 892:L3, 2020.

3. B.P. Abbott et al. Phys. Rev. Lett 119 161101, 2017.

4. R. Abbott et al. Astrophys. J. 896 (2020) no.2, L44 [arXiv:2006.12611 [astro-ph.HE]].

5. J. Abedi et al. Phys. Rev., D96(8):082004, 2017.

6. V. Cardoso et al. Phys. Rev. D 94 (2016) no.8, 084031 [arXiv:1608.08637 [gr-qc]].

7. A. Addazi, et al. Phys. Rev. Lett. 122 (2019) no.8, 081301 [arXiv:1810.10417 [gr-qc]].

8. E. Barausse et al. Class. Quant. Grav. 35 (2018) no.20, 20LT01 [arXiv:1805.08229 [gr-qc]].

9. V. Cardoso et al. Living Rev. Rel. 22 (2019) no.1, 4 [arXiv:1904.05363 [gr-qc]].

10. B. Chen et al. [arXiv:1902.08180 [gr-qc]].

11. P. Pani et al. Phys. Rev. D 92 (2015) no.2, 024010 [arXiv:1503.07365 [gr-qc]].

12. T. Binnington et al. Phys. Rev. D 80 (2009), 084018 [arXiv:0906.1366 [gr-qc]].

13. P. Pani et al. Phys. Rev. D 92 (2015) no.12, 124003 [arXiv:1509.02171 [gr-qc]].

14. V. Cardoso et al. Phys. Rev. D 95 (2017) no.8, 084014 [arXiv:1701.01116 [gr-qc]].

15. K. Yagi et al. Class. Quant. Grav. 33 (2016) no.9, 095005 [arXiv:1601.02171 [gr-qc]].

16. A. Le Tiec et al. [arXiv:2007.00214 [gr-qc]].

17. B. Guo, S. Hampton and S. D. Mathur, JHEP 07 (2018), 162 [arXiv:1711.01617 [hep-th]].

18. T. Harada et al. Phys. Rev. D 99 (2019) no.4, 044039 [arXiv:1811.05179 [gr-qc]].

19. A. Addazi et al. Eur. Phys. J. C 80 (2020) no.1, 36 [arXiv:1905.08734 [gr-qc]].

20. A. Paranjape et al. Phys. Rev. D 80 (2009), 044011 [arXiv:0906.1768 [gr-qc]].

21. M. Saravani et al. Int. J. Mod. Phys. D 23 (2015) no.13, 1443007 [arXiv:1212.4176 [hep-th]].

22. C. Barcelo et al. JHEP 02 (2011), 003 [arXiv:1011.5911 [gr-qc]].

23. N. Uchikata et al. Class. Quant. Grav. 33 (2016) no.2, 025005 [arXiv:1506.06485 [gr-qc]].

24. V. Cardoso et al. Class. Quant. Grav. 25 (2008), 195010 [arXiv:0808.1615 [gr-qc]].

25. V. Cardoso et al. Phys. Rev. D 77 (2008), 124044 [arXiv:0709.0532 [gr-qc]].

26. V. Cardoso et al. Phys. Rev. D 90 (2014) no.4, 044069 [arXiv:1406.5510 [gr-qc]].

27. Dmitri V. Fursaev. Phys. Part. Nucl., 36:81, 2005.

28. S. Mukohyama et al. Phys. Rev., D58:104005, 1998.

29. G. 't Hooft, Nucl. Phys. B 256 (1985), 727.

30. W. Israel, Phys.Lett.A 57 107, 1976.

31. W. H. Zurek et al. Phys. Rev. Lett. $54,2171,1985$.

32. S.A. Fulling et al. Phys. Rep. 152, 135, 1987.

33. J. R. Arenas S. et al. Nuovo Cim., B125:1223, 2010.

34. F. Pretorius et al. Phys. Rev., D57:6311, 1998.

35. R. Bousso, Rev. Mod. Phys. 74, 825, 2002. [arXiv:hep-th/0203101 [hep-th]].

36. J. D. Bekenstein Phys. Rev. D 23, 287, 1981.

37. C. Rovelli et al. Reality is Not What it Seems: The Journey to Quantum Gravity. Riverhead Books, 2017. ISBN 9780735213920.

38. W. Israel. Phys. Rev., 153:1388, Jan 1967.

39. J. R. Arenas S. et al. arXiv e-prints,.arXiv:1606.06786, 2016.

40. E. T. Akhmedov et al. Phys. Rev. D 93 (2016) no.2, 024029 [arXiv:1508.07500 [hep-th]].

41. W. Israel Nuovo Cim. B 44S10 (1966), 1 [erratum: Nuovo Cim. B 48 (1967), 463]

42. W. A. Rojas C. et al. [arXiv:1110.4058 [gr-qc]].

43. W. A. Rojas C. et al. [arXiv:1712.08724 [gr-qc]].

44. J. R. Oppenheimer et al. Phys. Rev. 56, 455, 1939. 
11 of 11

45. R. Penrose. Riv. Nuovo Cim., 1:252, 1969.

46. Thorne, K. S. et al. Black holes: The membrane paradigm 1986.

47. A. Ferrara et al. Mon. Not. Roy. Astron. Soc., 443(3):2410, 2014.

48. S. Basu et al. Astrophy. J., 879:(1): L3,2019. 